\title{
Option market making under inventory risk*
}

\author{
Sasha Stoikov ${ }^{\dagger} \quad$ Mehmet Sağlam ${ }^{\ddagger}$
}

February 15, 2009

\begin{abstract}
We propose a mean-variance framework to analyze the optimal quoting policy of an option market maker. The market maker's profits come from the bid-ask spreads received over the course of a trading day, while the risk comes from uncertainty in the value of his portfolio, or inventory. Within this framework, we study the impact of liquidity and market incompleteness on the optimal bid and ask prices of the option. First, we consider a market maker in a complete market, where continuous trading in a perfectly liquid underlying stock is allowed. In this setting, the market maker may remove all risk by Delta hedging, and the optimal quotes will depend on the option's liquidity, but not on the inventory. Second, we model a market maker who may not trade continuously in the underlying stock, but rather sets bid and ask quotes in the option and this illiquid stock. We find that the optimal stock and option quotes depend on the relative liquidity of both instruments as well as on the net Delta of the inventory. Third, we consider an incomplete market with residual risks due to stochastic volatility and large overnight moves in the stock price. In this setting, the optimal quotes depend on the liquidity of the option and on the net Vega and Gamma of the inventory.
\end{abstract}

Keywords: Delta, European options, Gamma, Inventory management, Liquidity, Market microstructure, Vega

\footnotetext{
*Stoikov acknowledges support from the Morgan Stanley Equity Market Microstructure Research Grant program. The views expressed herein are solely those of the authors and not those of Morgan Stanley. Both authors are grateful to Peter Bank, for some helpful comments.

${ }^{\dagger}$ Cornell University, Cornell Financial Engineering Manhattan, 55 Broad street, New York, NY, 10004, sashastoikov@gmail.com

${ }^{\ddagger}$ Columbia University, Graduate School of Business, 3022 Broadway, New York, NY 10027, ms3760@columbia.edu
} 


\section{Introduction}

We study the optimal bid and ask prices at which an options dealer, or market maker, sets his quotes. The market maker is committed to dynamically update bid and ask quotes in the options market, and is thus at the heart of the option pricing problem. By understanding the dealer's sources of risk and return, we may formulate his "optimal" quoting policy and thus gain insight in the dynamics of option prices. We capture fluctuations in the supply and demand for options by modeling the arrival intensity of orders as a function of how far the dealer's quotes are from the option's mid price. This approach leads to a quoting policy that depends on the risk of the dealer's inventory position as well as the liquidity of the stock and options market.

From an empirical perspective, we are inspired by papers describing the behavior of option dealers. For instance, in Garleanu, Pedersen and Poteshman (2006), the authors find that the net demand for options exerts pressure on option prices, when perfect replication is impossible. They derive the sensitivity of option prices with respect to net demand and they find that it depends on the standard "Greeks", Gamma and Vega. The price effect of risk due to discrete time hedging is dependent on the option's Gamma, while the price effect of risk due to stochastic volatility depends on Vega. A related insight may be found in Jameson and Wilhelm (1992), who show that Gamma and Vega contribute significantly to the bid-ask spread.

From a theoretical perspective, our stochastic control problem can be viewed as an inventory management problem, where the order flow depends on the bid and ask prices. This type of problem was first studied by Ho and Stoll (1981) and an empirical analysis was applied to the options market by Ho and Macris (1984). These authors show that if a dealer's goal is to maximize expected utility, he will adjust his quotes in response to inventory positions. As one would expect, when the dealer's inventory is positive, the quotes should be lowered and when the inventory is negative, the quotes should be raised. This simple risk-management mechanism helps the dealer keep inventory positions under control. Another related optimization problem is solved in Minina and Vellekoop (2008), which models an options trader whose risk is a function of the portfolio's Greeks.

There are some important differences between our model and those described above. First, we consider a dealer in the option market who provides liquidity with limit orders in the form of bid and ask quotes. If the underlying stock is illiquid, the dealer may also control his quotes on the stock. This 
approach allows us to model the relative liquidity of the option and stock markets and to determine the optimal policy for the dealer by solving the stochastic control problem. On the other hand, Garleanu, Pedersen and Poteshman (2006) consider the net demand pressure only on the options and derive the competitive equilibrium valuations of all options in the economy without focusing on optimal dealer policy or evolution of bid and ask quotes.

Second, we construct our model in a mean-variance framework in contrast to general expected utility model in Ho and Stoll (1981) and exponential utility model with consumption in Garleanu, Pedersen and Poteshman (2006). This type of objective has enjoyed a revival in the optimal order execution literature (see Almgren and Chriss (2001), Engle and Ferstenberg (2006)) and is a natural choice to address optimal market making strategies. Indeed, we find that a fast numerical procedure can be implemented to determine the optimal bid and ask prices, without resorting to asymptotic expansions, as is often the case in stochastic control problems of this nature.

Third, we choose an investment horizon of one day, and explicitly separate the problem into a day of trading, followed by an overnight move in the stock and option prices. The advantage of our approach is that we can capture the often observed fact that dealers tend to "go home flat" (see Hasbrouck (2007)). By framing our problem in a twenty-four-hour horizon, we find that the optimal dealer will move his quotes more aggressively towards the end of the day, so as to avoid keeping excessive inventory overnight.

In Section 2, we present the complete market version of our framework, where the option may be perfectly hedged by continuously trading in the underlying. The main result of this section is that the market maker's quotes do not depend on inventory. In Section 3, we solve a version of the model where the underlying is illiquid and the market maker updates quotes on both the stock and options market. We illustrate the fact that our mean-variance problem allows us to trace out the efficient frontier for a dealer, in analogy with the classic Markowitz approach. In Section 4, we introduce a version of the model where the dealer is subject to residual risks due to stochastic volatility and overnight moves in the stock price. We find that the bid and ask quoting policy depends on the net Gamma and Vega of the dealer. This affects long and short maturity options differently and we illustrate this by numerically computing the dealer's optimal policies. We present our conclusions in Section 5, and the proofs are all included in the appendix. 


\section{Complete market}

The market dynamics. We choose to model the stock price as a martingale under the historic probability measure, to reflect the idea that the dealer has no information or view on the future direction of the stock price. The dealer considers the market price to be the 'true' value of the stock at all times. On a technical level, the assumption that the stock price is a martingale will also turn out to be essential in establishing the dynamic programming principle for our mean-variance objective.

If we assume that the interest rate, $r$, equals zero, the stock and option price dynamics must also be martingales under the risk neutral measure to avoid arbitrage opportunities. Effectively, we choose a model where the dynamics under the historic and risk-neutral probability measures coincide in order to focus on the effects of a stochastic inventory on the dealer's quotes.

The continuous time dynamics of the stock mid price is given by

$$
d S_{t}=\sigma S_{t} d W_{t}
$$

The dealer makes markets in a European call option with maturity $T_{\text {mat }} \gg T$ and strike $K$, whose mid price follows

$$
d C(S, t)=\Theta_{t} d t+\Delta_{t} d S_{t}+\frac{1}{2} \Gamma_{t}\left(d S_{t}\right)^{2}=\Delta_{t} \sigma S_{t} d W_{t}
$$

where the function $C(S, t)$ is given by the Black Scholes formula and $\Theta_{t}, \Delta_{t}$ and $\Gamma_{t}$ are the standard greeks, Theta, Delta and Gamma, respectively.

The liquidity. The dealer will trade exclusively with limit orders in the form of bid and ask quotes "pegged" around the option's mid price,

$$
\begin{aligned}
& p_{t}^{b, o}=C_{t}-\epsilon_{t}^{b, o} \\
& p_{t}^{a, o}=C_{t}+\epsilon_{t}^{a, o}
\end{aligned}
$$

He stands ready to trade one option lot at the prices above, where $\epsilon_{t}^{a, o}$ and $\epsilon_{t}^{b, o}$ are the premiums obtained by the market-maker. If we only admit premiums that are greater than zero, these prices 
represent an improvement over the mid price of the option. Note that as long as the mid price specifications are arbitrage free, as is the case in (1) and (2), the dealer's quotes cannot be arbitraged. The bid and ask quotes, or equivalently the premiums, $\epsilon_{t}^{a, o}$ and $\epsilon_{t}^{b, o}$, indirectly influence the inventory held by the market maker, since they affect the arrival rates of orders. We will let $\lambda_{o}(\epsilon)$ be the Poisson arrival rate of option orders. $\lambda_{o}(\epsilon)$ needs to obey certain restrictions for the existence of an optimal policy.

The dealer's state variables and controls. The dealer may continuously control the wealth in stock $\pi_{t}$ and the bid and ask premiums $\epsilon_{t}^{a, o}$ and $\epsilon_{t}^{b, o}$ on the option. The number of options bought and sold before time $t$, respectively $N_{t}^{b, o}$ and $N_{t}^{a, o}$, are Markov modulated Poisson processes with rates $\lambda\left(\epsilon_{t}^{b, o}\right)$ and $\lambda\left(\epsilon_{t}^{a, o}\right)$.

$$
q_{t}^{o}=N_{t}^{b, o}-N_{t}^{a, o}
$$

is the inventory in options.

The marked-to-market wealth $X_{t}$ is given by

$$
X_{t}=\pi_{t}^{0}+\pi_{t}+q_{t}^{o} C_{t}
$$

where $\pi_{t}^{0}$ is the dollar amount in cash. It follows that

$$
d X_{t}=\sigma \pi_{t} d W_{t}+\epsilon_{t}^{a, o} d N_{t}^{a, o}+\epsilon_{t}^{b, o} d N_{t}^{b, o}+q_{t}^{o} d C_{t}
$$

We may decompose this wealth process into the returns obtained from transactions

$$
d Z_{t}=\epsilon_{t}^{a, o} d N_{t}^{a, o}+\epsilon_{t}^{b, o} d N_{t}^{b, o}
$$

and the inventory value

$$
d I_{t}=\sigma \pi_{t} d W_{t}+q_{t}^{o} d C_{t}
$$

The objective. We consider the stochastic control problem of a dealer who sets bid and ask prices 
throughout the trading day. The value function is

$$
v\left(Z_{0}, S_{0}, q_{0}^{o}, 0\right)=\max _{\pi_{t}, \epsilon_{t}^{a, o}, \epsilon_{t}^{b, o}, 0 \leq t \leq T}\left(\mathbb{E}\left[Z_{T}\right]-\gamma \operatorname{Var}\left[I_{T}\right]\right)
$$

where $\gamma$ is dealer's parameter of risk aversion. The dealer wishes to maximize profit from transactions, with a penalty proportional to the variance of cumulated inventory risk.

The following result illustrates that if the options can be dynamically replicated with the stock, the quoting policy is independent of the inventory.

Theorem 1. The optimal dollar amount of stock in (3) is given by

$$
\pi_{t}=-S_{t} \Delta_{t} q_{t}^{o}
$$

and optimal quoting policy is given by the implicit equations

$$
\epsilon_{t}^{a, o}=-\frac{\lambda_{o}\left(\epsilon_{t}^{a, o}\right)}{\lambda_{o}^{\prime}\left(\epsilon_{t}^{a, o}\right)}
$$

and

$$
\epsilon_{t}^{b, o}=-\frac{\lambda_{o}\left(\epsilon_{t}^{b, o}\right)}{\lambda_{o}^{\prime}\left(\epsilon_{t}^{b, o}\right)}
$$

Note that optimal premiums are well-defined if the revenue function, $R(\epsilon)=\epsilon \lambda_{o}(\epsilon)$, has a maximizer. Using the necessary conditions for optimality, we need

$$
\epsilon \lambda_{o}^{\prime \prime}(\epsilon)+2 \lambda_{o}^{\prime}(\epsilon) \leq 0
$$

Therefore, an appropriate choice for $\lambda_{o}(\epsilon)$ would be a decreasing linear function. In the sequel, we let

$$
\lambda_{o}(\epsilon)= \begin{cases}C-D \epsilon & \text { if } 0 \leq \epsilon<C / D \\ 0 & \text { otherwise }\end{cases}
$$

be the Poisson arrival rates of option orders. This choice of decreasing function with respect to $\epsilon$ also coincides with the empirical fact that the closer the quotes are to the mid price, the higher the 
probability of their execution in a given time interval. We introduce cut-offs to capture the fact that there is a minimum spread that must be quoted by the market maker at all times.

In the next section, we consider a setting where the underlying stock is illiquid and the dealer makes markets in both the stock and the option.

\section{Incomplete market due to illiquidity in the underly- ing stock}

Illiquidity in the stock price may be modeled as a proportional transaction cost, as in Davis, Panas and Zariphopoulou (1993). In such models, the agent crosses the bid-ask spread in order to reduce risk in a static options position, and the state space may be decomposed into a trade and a no-trade region. Our approach here will be to model the market maker's transactions in stocks in the same way as the options transactions described in the previous section. In other words, the dealer will now

control the premiums that the dealer charges around the option mid price, $\epsilon_{t}^{a, o}$ and $\epsilon_{t}^{b, o}$, as well as around the stock mid price, $\epsilon_{t}^{a, s}$ and $\epsilon_{t}^{b, s}$ where

$$
\begin{gathered}
p_{t}^{b, s}=S_{t}-\epsilon_{t}^{b, s} \\
p_{t}^{a, s}=S_{t}+\epsilon_{t}^{a, s} .
\end{gathered}
$$

If we assume arrival rates for the stock transactions to be linear with respect to premiums as in the case of option trades,

$$
\lambda_{s}(\epsilon)= \begin{cases}A-B \epsilon & \text { if } 0 \leq \epsilon<A / B \\ 0 & \text { otherwise }\end{cases}
$$

we may adjust the constants, $A, B, C$ and $D$, to capture the relative liquidity of the stock and option markets.

The wealth now follows

$$
d X_{t}=\epsilon_{t}^{a, s} d N_{t}^{a, s}+\epsilon_{t}^{b, s} d N_{t}^{b, s}+q_{t}^{s} d S_{t}+\epsilon_{t}^{a, o} d N_{t}^{a, o}+\epsilon_{t}^{b, o} d N_{t}^{b, o}+q_{t}^{o} d C_{t}
$$


We may decompose this wealth process into the returns obtained from transactions

$$
d Z_{t}=\epsilon_{t}^{a, s} d N_{t}^{a, s}+\epsilon_{t}^{b, s} d N_{t}^{b, s}+\epsilon_{t}^{a, o} d N_{t}^{a, o}+\epsilon_{t}^{b, o} d N_{t}^{b, o}
$$

and the inventory risk

$$
d I_{t}=q_{t}^{s} d S_{t}+q_{t}^{o} d C_{t} .
$$

We now consider the stochastic control problem of a dealer who sets bid and ask prices on the stock and the option throughout the trading day. The value function is

$$
v\left(Z_{0}, S_{0}, q_{0}^{s}, q_{0}^{o}, 0\right)=\max _{\epsilon_{t}^{a, s}, \epsilon_{t}^{b, s}, \epsilon_{t}^{a, o}, \epsilon_{t}^{b, o}, 0 \leq t \leq T} \mathbb{E}\left[Z_{T}\right]-\gamma \operatorname{Var}\left[I_{T}\right]
$$

The discrete time problem. The difficulty in solving problem (6) is that we may no longer reduce the variance term to zero, by dynamically adjusting the stock position, as in the previous section. Our approach will be to discretize the problem to obtain a numerical solution.

We divide the trading day into $n$ sessions $0=t_{0}<t_{1}<\ldots<t_{n}$. At the beginning of every session, the dealer sets bid and ask quotes on the stock $\left(p_{i}^{b, s}\right.$ and $\left.p_{i}^{a, s}\right)$ and on the option $\left(p_{i}^{b, o}\right.$ and $\left.p_{i}^{a, o}\right)$ for $0 \leq i \leq n-1$, and waits for market orders to arrive.

At the end of the $n^{\text {th }}$ session, the market is closed and no trading occurs in the interval $\left(t_{n}, T\right]$. There is an overnight move in the asset prices, where $T \triangleq t_{n+1}$ represents the beginning of the following trading day. The explicit modeling of this overnight move is an important ingredient of our modeling. Because the dealer is unable to trade in the interval $\left(t_{n}, T\right]$, the bid and ask quotes will turn out to be more sensitive to the inventory positions as the end of the day approaches. This feature differs from the inventory models of Ho and Stoll (1981), where the sensitivity of the quotes with respect to inventory decreases as the horizon $T$ approaches.

Conditional probabilities associated with transactions returns and changes in inventory can be 
approximated up to terms of order $o(\Delta t)$ by

$$
\begin{array}{lll}
P\left(\Delta Z_{i}=0 \mid \mathcal{F}_{i}\right) & =P\left(\Delta q_{i}^{s}=0, \Delta q_{i}^{o}=0\right) & =1-\left(\lambda_{s}\left(\epsilon_{i}^{b, s}\right)+\lambda_{s}\left(\epsilon_{i}^{a, s}\right)+\lambda_{o}\left(\epsilon_{i}^{b, o}\right)+\lambda_{o}\left(\epsilon_{i}^{a, o}\right)\right) \Delta t \\
P\left(\Delta Z_{i}=\epsilon_{i}^{b, s} \mid \mathcal{F}_{i}\right) & =P\left(\Delta q_{i}^{s}=1, \Delta q_{i}^{o}=0\right) & =\lambda_{s}\left(\epsilon_{i}^{b, s}\right) \Delta t \\
P\left(\Delta Z_{i}=\epsilon_{i}^{a, s} \mid \mathcal{F}_{i}\right) & =P\left(\Delta q_{i}^{s}=-1, \Delta q_{i}^{o}=0\right) & =\lambda_{s}\left(\epsilon_{i}^{a, s}\right) \Delta t \\
P\left(\Delta Z_{i}=\epsilon_{i}^{b, o} \mid \mathcal{F}_{i}\right) & =P\left(\Delta q_{i}^{s}=0, \Delta q_{i}^{o}=1\right) & =\lambda_{o}\left(\epsilon_{i}^{b, o}\right) \Delta t \\
P\left(\Delta Z_{i}=\epsilon_{i}^{a, o} \mid \mathcal{F}_{i}\right) & =P\left(\Delta q_{i}^{s}=0, \Delta q_{i}^{o}=-1\right) & =\lambda_{o}\left(\epsilon_{i}^{a, o}\right) \Delta t
\end{array}
$$

where $\mathcal{F}_{i}$ denotes the available information at time $t_{i}$.

If we use the convention that trades happen immediately after time $t_{i}$, the change in the market value of the inventory is given by

$$
\Delta I_{i} \triangleq q_{i+1}^{s} \Delta S_{i}+q_{i+1}^{o} \Delta C_{i}
$$

and we assume without loss of generality that $I_{0}=0$. Note that since no trading occurs in the period $\left(t_{n}, T\right), \Delta Z_{n} \triangleq Z_{T}-Z_{n}=0$ and

$$
\Delta I_{n} \triangleq I_{T}-I_{n}=q_{n}^{s}\left(S_{T}-S_{n}\right)+q_{n}^{o}\left(C_{T}-C_{n}\right)
$$

Since the increments $\Delta I_{i}$ are independent, the discretized version of our problem may be written as

$$
v\left(Z_{0}, S_{0}, q_{0}^{s}, q_{0}^{o}, t_{0}\right)=\max _{\epsilon_{i}^{a, s}, \epsilon_{i}^{b, s}, \epsilon_{i}^{a, o}, \epsilon_{i}^{b, o}, 0 \leq i \leq n-1}\left(\mathbb{E}\left[Z_{T}\right]-\gamma \sum_{i=0}^{n} \operatorname{Var}\left[\Delta I_{i}\right]\right)
$$

\section{A One-period model}

Let us first solve the one-period version of our model. To keep notation coherent with the rest of the paper, we consider the dealer's problem at the beginning of the last trading session, assuming that he may only trade in the interval $\left(t_{n-1}, t_{n}\right)$. The dealer chooses bid and ask quotes at time $t_{n-1}$, defined through the controls $\epsilon_{n-1}^{a, s}, \epsilon_{n-1}^{b, s}, \epsilon_{n-1}^{a, o}$ and $\epsilon_{n-1}^{b, o}$. These quotes influence the probabilities of the four possible transactions (see $(7))$ over the time interval $\left(t_{n-1}, t_{n}\right)$. We initially assume that the stock and the option prices do not change in the last trading session, i.e., $S_{n}=S_{n-1}$ and $C_{n}=C_{n-1}$, but may change in the overnight interval $\left(t_{n}, T\right)$. 
The dealer's objective is to maximize expected marked to market wealth at time $T$, with a penalty term that is proportional to the variance of the change in value of the inventory. This mean-variance objective therefore strikes a balance between the desire to maximize the marked to market profits made from the bid-ask spread and the risk associated with changes in market prices.

The value function can be written as

$$
v\left(Z_{n-1}, S_{n-1}, q_{n-1}^{s}, q_{n-1}^{o}, t_{n-1}\right)=\max _{\epsilon_{n-1}^{a, s}, \epsilon_{n-1}^{b, s}, \epsilon_{n-1}^{a, o}, \epsilon_{n-1}^{b, o}}\left(\mathbb{E}\left[Z_{T} \mid \mathcal{F}_{n-1}\right]-\gamma \operatorname{Var}\left[\Delta I_{n} \mid \mathcal{F}_{n-1}\right]\right)
$$

The dealer's objective is to determine the optimal bid and ask quotes on the stock and options market.

Theorem 2. The optimal policy for the dealer is given by

$$
\begin{aligned}
\epsilon_{n-1}^{a, s} & =\max \left(0, \min \left(\frac{A}{B}, \frac{A}{2 B}-\gamma \sigma^{2}\left(T-t_{n}\right) S_{n}^{2}\left(q_{n-1}^{s}+q_{n-1}^{o} \Delta_{n}-\frac{1}{2}\right)\right)\right) \\
\epsilon_{n-1}^{b, s} & =\max \left(0, \min \left(\frac{A}{B}, \frac{A}{2 B}+\gamma \sigma^{2}\left(T-t_{n}\right) S_{n}^{2}\left(q_{n-1}^{s}+q_{n-1}^{o} \Delta_{n}+\frac{1}{2}\right)\right)\right) \\
\epsilon_{n-1}^{a, o} & =\max \left(0, \min \left(\frac{C}{D}, \frac{C}{2 D}-\gamma \sigma^{2}\left(T-t_{n}\right) S_{n}^{2} \Delta_{n}\left(q_{n-1}^{s}+q_{n-1}^{o} \Delta_{n}-\frac{1}{2} \Delta_{n}\right)\right)\right) \\
\epsilon_{n-1}^{b, o} & =\max \left(0, \min \left(\frac{C}{D}, \frac{C}{2 D}+\gamma \sigma^{2}\left(T-t_{n}\right) S_{n}^{2} \Delta_{n}\left(q_{n-1}^{s}+q_{n-1}^{o} \Delta_{n}+\frac{1}{2} \Delta_{n}\right)\right)\right)
\end{aligned}
$$

In contrast to the complete market setting, the optimal premiums are piecewise linear functions of inventory. In the risk neutral case where $\gamma=0$, the objective is to maximize terminal wealth and the optimal premiums are

$$
\begin{aligned}
& \epsilon_{n-1}^{a, s}=\epsilon_{n-1}^{b, s}=\frac{A}{2 B} \\
& \epsilon_{n-1}^{a, o}=\epsilon_{n-1}^{b, o}=\frac{C}{2 D}
\end{aligned}
$$

regardless of inventory. If the dealer is risk-averse, i.e. $\gamma>0$, he adjusts or "tilts" all his quotes away from the revenue maximizing solution by an amount proportional to $q_{n-1}^{s}+q_{n-1}^{o} \Delta_{n}$ which is the net Delta.

\section{B Multi-period model}

We now consider a multi-period model where the stock price does not move during the day and the only inventory risk comes from the possibility of an overnight move. This simplifies the problem, since most of the variance terms in (10) drop out and the only source of uncertainty during the trading day 
comes from the transactions. In this setting the stock and option premiums $\epsilon^{a, s}, \epsilon^{b, s}, \epsilon^{a, o}$ and $\epsilon^{b, o}$ can be computed recursively as functions of time and inventory (see Theorem 3.2).

The stock price is fixed at $S_{i}=S$ for $i \leq n$ and

$$
S_{T}=S+\sigma S\left(W_{T}-W_{t_{n}}\right)
$$

Likewise, the option price remains constant at $C_{i}=C$ throughout the day and then becomes

$$
C_{T}=C+\sigma S \Delta_{n}\left(W_{T}-W_{t_{n}}\right)
$$

The objective (10) simplifies to

$$
v\left(Z_{0}, S_{0}, q_{0}^{s}, q_{0}^{o}, t_{0}\right)=\max _{\epsilon_{i}^{a, s}, \epsilon_{i}^{b, s}, \epsilon_{i}^{a, o}, \epsilon_{i}^{b, o}, 0 \leq i \leq n-1}\left(\mathbb{E}\left[Z_{T}\right]-\gamma \operatorname{Var}\left[\Delta I_{n}\right]\right)
$$

If we define

$$
v_{j}\left(Z_{j}, q_{j}^{s}, q_{j}^{o}\right)=\max _{\epsilon_{i}^{a, s}, \epsilon_{i}^{b, s}, \epsilon_{i}^{a, o}, \epsilon_{i}^{b, o}, j \leq i \leq n-1}\left(\mathbb{E}\left[Z_{T} \mid \mathcal{F}_{j}\right]-\gamma \operatorname{Var}\left[\Delta I_{n} \mid \mathcal{F}_{j}\right]\right)
$$

we may use the tower property of conditional expectations and the conditional variance formula

$$
\begin{aligned}
\mathbb{E}\left[Z_{T} \mid \mathcal{F}_{i}\right] & =\mathbb{E}\left[\mathbb{E}\left[Z_{T} \mid \mathcal{F}_{i+1}\right] \mid \mathcal{F}_{i}\right] \\
\operatorname{Var}\left[\Delta I_{n} \mid \mathcal{F}_{i}\right] & =\mathbb{E}\left[\operatorname{Var}\left[\Delta I_{n} \mid \mathcal{F}_{i+1}\right] \mid \mathcal{F}_{i}\right]+\operatorname{Var}\left[\mathbb{E}\left[\Delta I_{n} \mid \mathcal{F}_{i+1}\right] \mid \mathcal{F}_{i}\right] \\
& =\mathbb{E}\left[\operatorname{Var}\left[\Delta I_{n} \mid \mathcal{F}_{i+1}\right] \mid \mathcal{F}_{i}\right]
\end{aligned}
$$

to obtain the dynamic programming principle

$$
v_{i}\left(Z_{i}, q_{i}^{s}, q_{i}^{o}\right)=\max _{\epsilon_{i}^{a, s}, \epsilon_{i}^{b, s}, \epsilon_{i}^{a, o}, \epsilon_{i}^{b, o}} \mathbb{E}\left[v_{i+1}\left(Z_{i+1}, q_{i+1}^{s}, q_{i+1}^{o}\right) \mid \mathcal{F}_{i}\right]
$$

Note that (14) depends on the fact that the stock price is a martingale.

The computational task of building a non-recombining pentanomial tree to solve such a problem seems daunting. Fortunately, the value function $v_{i}$ can be expressed as the linear combination of (i) the marked to market wealth $Z_{i}$ accumulated until time $i$ and (ii) a function of inventory in net Delta. It follows that the optimal bid and ask premiums are piecewise linear in net Delta. This is summarized 

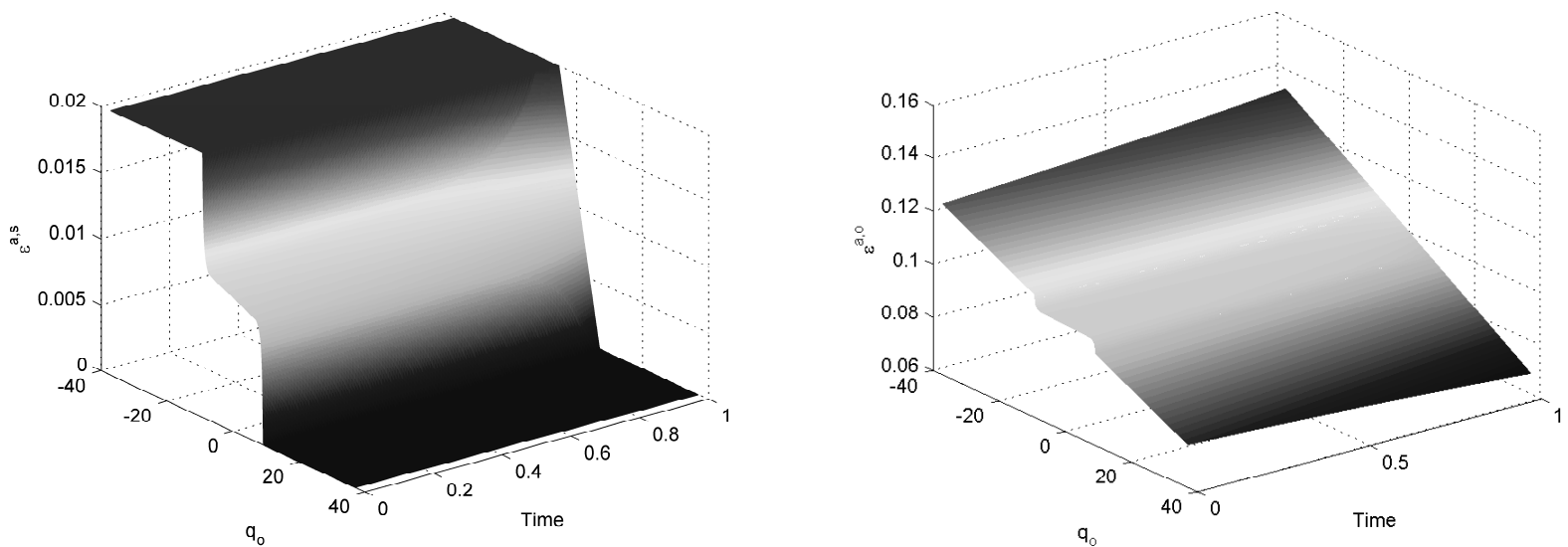

Figure 1: Optimal quoting policy of a dealer with low risk aversion in the case of no intraday movement in the stock price. As net inventory increases ( $q_{o}$ in this case), the premiums $\epsilon_{i}^{a, s}$ and $\epsilon_{i}^{a, o}$ decrease. The rate of decrease $\left(m_{i}\right)$ is much higher at the end of the day. $\left(\gamma=0.006, q^{s}=0, A=40, B=2000, C=40, D=200, S=100, K=100, \sigma=0.01, n=100, t_{0}=0\right.$, $\left.t_{n}=1, T=1.5, T_{m a t}=400.\right)$

in the following theorem.

Theorem 3. The optimal bid and ask premiums at time $t_{i}$ are given by

$$
\begin{aligned}
\epsilon_{i}^{a, s} & =\max \left(0, \min \left(\frac{A}{B}, \frac{A}{2 B}+m_{i+1}\left(q_{i}^{s}+\Delta_{n} q_{i}^{o}-\frac{1}{2}\right)\right)\right) \\
\epsilon_{i}^{b, s} & =\max \left(0, \min \left(\frac{A}{B}, \frac{A}{2 B}-m_{i+1}\left(q_{i}^{s}+\Delta_{n} q_{i}^{o}+\frac{1}{2}\right)\right)\right) \\
\epsilon_{i}^{a, o} & =\max \left(0, \min \left(\frac{C}{D}, \frac{C}{2 D}+m_{i+1} \Delta_{n}\left(q_{i}^{s}+\Delta_{n} q_{i}^{o}-\frac{1}{2} \Delta_{n}\right)\right)\right) \\
\epsilon_{i}^{a, o} & =\max \left(0, \min \left(\frac{C}{D}, \frac{C}{2 D}-m_{i+1} \Delta_{n}\left(q_{i}^{s}+\Delta_{n} q_{i}^{o}+\frac{1}{2} \Delta_{n}\right)\right)\right)
\end{aligned}
$$

where $m_{i}$ is the slope coefficient of the "tilting" and is calculated with the following recursion

$$
m_{i}=m_{i+1}+\Delta t\left(B I_{i}+D \Delta_{n}^{2} J_{i}\right) m_{i+1}^{2}
$$

with the terminal condition $m_{n}=-\gamma \sigma^{2} S^{2}\left(T-t_{n}\right)$ and the auxiliary variables, $I_{i}=\left(\mathbb{1}_{\left\{0<\epsilon_{i}^{a, s}<\frac{A}{B}\right\}}+\mathbb{1}_{\left\{0<\epsilon_{i}^{b, s}<\frac{A}{B}\right\}}\right)$ and $J_{i}=\left(\mathbb{1}_{\left\{0<\epsilon_{i}^{a, o}<\frac{C}{D}\right\}}+\mathbb{1}_{\left\{0<\epsilon_{i}^{b, o}<\frac{C}{D}\right\}}\right)$.

In Figures 1 and 2, we illustrate the extent to which the amount of options in inventory, $q^{o}$, affects the ask premium on the stock, $\epsilon_{i}^{a, s}$ and on the option $\epsilon_{i}^{a, o}$, for two different levels of risk aversion $\gamma=0.006$ and $\gamma=0.1$. Note that we are setting $q^{s}=0$. We chose values of the liquidity parameters ( $A=40, B=2000, C=40, D=200$ ) that lead to spreads on the stock and the option of 2 and 20 cents respectively. Figure 1 illustrates that as $q^{o}$ increases, the premiums $\epsilon_{i}^{a, s}$ and $\epsilon_{i}^{a, o}$ decrease, 

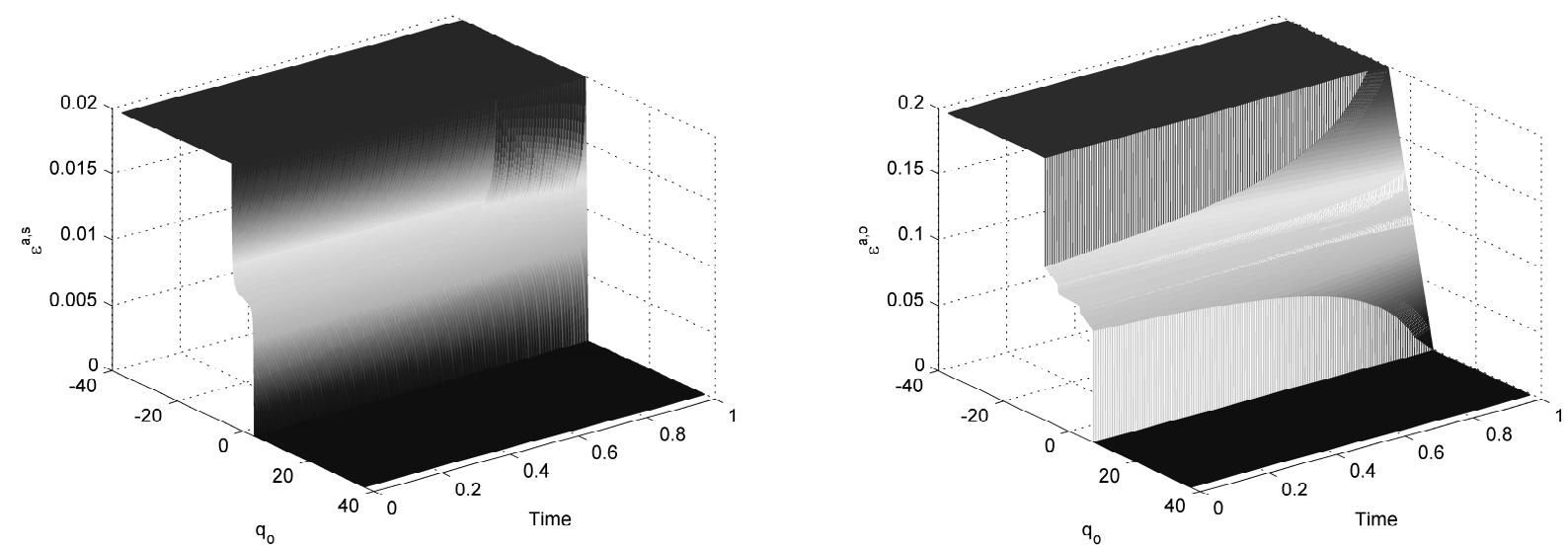

Figure 2: Optimal quoting policy of a dealer with high risk aversion in the case of no intraday movement in the stock price. Compared to Figure 1, the tilting in the quotes is much more aggressive. $\left(\gamma=0.1, q^{s}=0, A=40, B=2000, C=40\right.$, $D=200, S=100, K=100, \sigma=0.01, n=100, t_{0}=0, t_{n}=1, T=1.5, T_{\text {mat }}=400$.)

indicating that the ask quote moves closer to the market mid price. This effect is most dramatic on the stock quotes, where an inventory of 10 options causes the dealer to lower his ask quote aggressively to the mid price of the stock (i.e. $\epsilon_{i}^{a, s}=0$ ). In Figure 2, we see that for a very risk-averse dealer $(\gamma=0.1)$, the policy on the stock is essentially in two states: an aggressive sell state when $\epsilon_{i}^{a, s}=0$ and a no-trade state when $\epsilon_{i}^{a, s}=0.02$.

Also worthy of note is the time-of-the-day effect: near the end of the day $\left(t_{n} \approx 1\right)$, the ask quotes are more sensitive to small changes in inventory, than at the beginning of the day $\left(m_{n} \gg m_{0}\right)$. This reflects the fact that at the beginning of the day, the dealer has more chances to trade in and out of a position, while at the end of the day, he is most likely to get stuck with his marginal inventory.

For each level of risk aversion, there is an optimal quoting strategy, given by the premiums $\epsilon_{i}^{a, s}$, $\epsilon_{i}^{b, s}, \epsilon_{i}^{a, o}$ and $\epsilon_{i}^{b, o}$. By simulating 1000 days with initial inventory equal to zero, we obtain an efficient frontier (see Figure 3). This frontier describes the tradeoff between the expected daily profit and the standard deviation of the inventory value, for various levels of risk aversion. The discontinuities in the frontier are due to our particular modeling of the functions $\lambda_{s}$ and $\lambda_{o}$ in (4) and (5). The profit maximizing point $(\gamma=0)$ is obtained by a risk neutral dealer whose bid-ask quotes are symmetric around the mid price, regardless of inventory.

We identify two qualitatively different regions of the frontier, of which our choices of $\gamma=0.006$ and $\gamma=0.1$ are representative. The low risk aversion dealer, $\gamma=0.006$ essentially treats the stock and the 


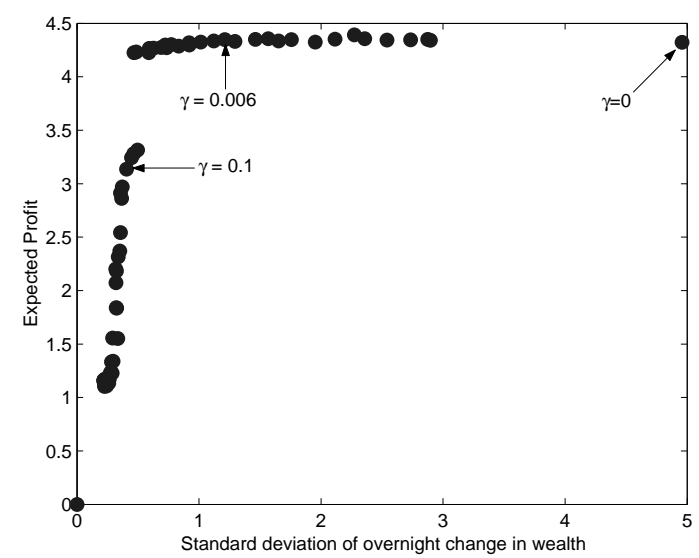

Figure 3: Efficient frontier. There are qualitatively two different regions of the frontier. The low risk aversion dealer, $\gamma=0.006$, treats the stock and the option as two similar instruments by slightly moving quotes on the stock and the option. The highly risk-averse dealer, $\gamma=0.1$, uses the stock mainly as a hedging instrument by aggressively moving the stock quotes and abandons his role as a market maker in the stock. $(A=40, B=2000, C=40, D=200, S=100, K=100, \sigma=0.01$, $n=100, t_{0}=0, t_{n}=1, T=1.5, T_{m a t}=400$.)

option as two similar instruments by slightly moving quotes on the stock and the option, for moderate levels of inventory (as illustrated in Figure 1). The highly risk-averse dealer, $\gamma=0.1$, uses the stock mainly as a hedging instrument by aggressively moving the stock quotes when his Delta is non-zero (as illustrated in Figure 2). This type of dealer would be likely to hedge options very frequently, even at the cost of crossing the spread, essentially abandoning his role as a market maker in the stock. We will in fact revisit dealers of this type in Section 4, by modeling a dealer who only makes markets in the options and delta-hedges his position at every time-step. This will require a more sophisticated modeling of the option dynamics and will highlight the role of higher order risks, Gamma and Vega.

\section{Incomplete market due to stochastic volatility and jump risk}

In this section, we introduce Gamma risk, by considering the risk due to an overnight jump in the stock price, and Vega risk, by directly modeling the stochastic implied volatility of the option. However, we also simplify the problem, by abandoning the dealer's control of the bid and ask quotes (or equivalently the controls $\epsilon^{a, s}$ and $\epsilon^{b, s}$ ) and assuming that the delta of his option inventory is set to zero at the beginning of every trading period $t_{i}$. 
The market dynamics. The continuous time dynamics of the stock mid price is given by

$$
d S_{t}=\sigma_{t} S_{t} d W_{t}
$$

where the volatility $\sigma_{t}$ is stochastic. Here we follow the approach of Schönbucher (1999) by directly modeling the implied volatility of the option as

$$
d \hat{\sigma}=\alpha d W_{t}^{1}
$$

where the Brownian motions $W_{t}$ and $W_{t}^{1}$ are assumed to be independent for the sake of simplicity.

The dealer makes markets in a European call option with maturity $T_{m a t}$ and strike $K$, whose mid price follows

$$
d C(S, t)=\Theta_{t} d t+\Delta_{t} d S_{t}+\frac{1}{2} \Gamma_{t}\left(d S_{t}\right)^{2}+C_{\sigma} d \hat{\sigma}_{t}+\frac{1}{2} C_{\sigma \sigma}\left(d \hat{\sigma}_{t}\right)^{2}
$$

where the function $C(S, t)$ is given by the Black Scholes formula and $\Theta_{t}, \Delta_{t}$ and $\Gamma_{t}$ are the standard greeks. Schönbucher (1999) shows that for the choice (15), the stochastic volatility of the stock must be related to the implied volatility through the relation

$$
\sigma_{t}^{2}=\hat{\sigma}_{t}^{2}-\frac{\alpha^{2}}{\hat{\sigma}_{t}^{2}}\left(\left(\ln \left(\frac{S}{K}\right)\right)^{2}-\frac{1}{4}\left(T_{m a t}-t\right)^{2} \hat{\sigma}_{t}^{4}\right)
$$

in order to satisfy

$$
0=\left(\Theta_{t}+\frac{1}{2} \hat{\sigma}_{t}^{2} S_{t}^{2} \Gamma_{t}\right) d t+\left(\frac{1}{2}\left(\sigma_{t}^{2}-\hat{\sigma}_{t}^{2}\right) S_{t}^{2} \Gamma_{t}+\frac{1}{2} C_{\sigma \sigma} \alpha^{2}\right) d t
$$

and thus ensure absence of arbitrage opportunities. Our expression for the change in option value, thus, becomes

$$
\Delta C_{i}=\Delta_{i} \sigma_{i} S_{i} u \sqrt{\Delta t}+\frac{1}{2} \Gamma_{i} \sigma_{i}^{2} S_{i}^{2}\left(u^{2}-1\right) \Delta t+C_{\sigma} \alpha \eta \sqrt{\Delta t}
$$

where $u$ and $\eta$ are standard normal random variables. The second term is a standard second order approximation (see Boyle and Emanuel (1980)), which captures the risk of discrete hedging. The third term captures the risk of a stochastic implied volatility. Using (16), the relationship between Gamma 
and Vega

$$
C_{\sigma}=\Gamma S^{2} \sigma\left(T_{m a t}-t\right)
$$

and assuming that the options are delta-hedged at every time step, i.e., $q_{i}^{s}=-q_{i}^{o} \Delta_{i}$, we may use (8) to obtain

$$
\Delta I_{i}=q_{i+1}^{o}\left(\frac{1}{2} \Gamma_{i} \sigma_{i}^{2} S_{i}^{2}\left(u^{2}-1\right) \Delta t+\Gamma_{i} \sigma_{i} \alpha S_{i}^{2}\left(T_{m a t}-t_{i}\right) \eta \sqrt{\Delta t}\right)
$$

and

$$
\Delta I_{n}=q_{n}^{o}\left(\frac{1}{2} \Gamma_{n} \sigma_{n}^{2} S_{n}^{2}\left(u^{2}-1\right) \Delta t+\Gamma_{n} \sigma_{n} \alpha S_{n}^{2}\left(T_{m a t}-t_{n}\right) \eta \sqrt{\Delta t}\right)
$$

Expression (18) highlights the fact that for long maturity options the stochastic volatility contribution is likely to dominate, while for short maturity options, the risk contribution of discrete hedging will be more important.

The advantage of directly modeling the implied volatility is that it is observable and that statistics on its volatility $\alpha$ can readily be obtained. Moreover, this approach can be generalized to a model of the entire volatility surface (see Cont and Da Fonseca (2002)).

\section{A One-period model}

We initially assume that the stock and the option prices and implied volatility do not change in the last trading session, i.e., $S_{n}=S_{n-1}, C_{n}=C_{n-1}$ and $\sigma_{n}=\sigma_{n-1}$, but may change in the overnight interval $\left(t_{n}, T\right)$. In this framework, the value function can be written as

$$
v\left(Z_{n-1}, S_{n-1}, q_{n-1}^{s}, q_{n-1}^{o}, t_{n-1}\right)=\max _{\substack{a, o \\ \epsilon_{n-1}, \epsilon_{n-1}^{b o}}} \mathbb{E}\left[Z_{T} \mid \mathcal{F}_{n-1}\right]-\gamma \operatorname{Var}\left[\Delta I_{n} \mid \mathcal{F}_{n-1}\right]
$$

The dealer's objective is to determine the optimal bid and ask quotes on the options market. Notice

that the value function depends on two controls $\epsilon_{i}^{a, o}$ and $\epsilon_{i}^{b, o}$, but the inventory risk now depends on higher order terms given in (18) and (19). 
Theorem 4. The optimal policy for the dealer is given by

$$
\begin{aligned}
& \epsilon_{n-1}^{a, o}=\max \left(0, \min \left(\frac{C}{D}, \frac{C}{2 D}-\gamma k\left(q_{n-1}^{o}-\frac{1}{2}\right)\right)\right) \\
& \epsilon_{n-1}^{b, o}=\max \left(0, \min \left(\frac{C}{D}, \frac{C}{2 D}+\gamma k\left(q_{n-1}^{o}+\frac{1}{2}\right)\right)\right)
\end{aligned}
$$

where $k=\left(\frac{1}{2} \sigma_{n}^{2}\left(T-t_{n}\right)+\alpha^{2}\left(T_{m a t}-t_{n}\right)^{2}\right) \Gamma_{n}^{2} S_{n}^{4} \sigma_{n}^{2}\left(T-t_{n}\right)$

As in the case of illiquidity in the underlying stock, a risk-neutral dealer, i.e. $\gamma=0$, sets optimal premiums equal to the revenue maximizing solution

$$
\epsilon_{n-1}^{a, o}=\epsilon_{n-1}^{b, o}=\frac{C}{2 D}
$$

regardless of inventory. However, in contrast to the that case, a risk-averse dealer, i.e. $\gamma>0$, "tilts" his quotes away from the revenue maximizing solution by an amount proportional to the net option position, $q_{n-1}^{o}$ where the constant of proportionality is a function of Gamma and volatility of implied volatility.

\section{B Multi-period model}

Much like in Section 3.2, we may use the dynamic programming principle to solve the multi-period model in the setting with no intraday movement. The value function simplifies to

$$
v\left(Z_{0}, S_{0}, q_{0}^{s}, q_{0}^{o}, t_{0}\right)=\max _{\epsilon_{i}^{a, o}, \epsilon_{i}^{b, o}, 0 \leq i \leq n-1} \mathbb{E}\left[Z_{T}\right]-\gamma \operatorname{Var}\left[\Delta I_{n}\right]
$$

and can be computed by applying the following result, analogous to Theorem 3.2.

Theorem 5. The optimal bid and ask premiums at time $t_{i}$ are given by

$$
\begin{aligned}
\epsilon_{i}^{a, o} & =\max \left(0, \min \left(\frac{C}{D}, \frac{C}{2 D}+m_{i+1} q_{i}^{o}-\frac{1}{2} m_{i+1}\right)\right) \\
\epsilon_{i}^{b, o} & =\max \left(0, \min \left(\frac{C}{D}, \frac{C}{2 D}-m_{i+1} q_{i}^{o}-\frac{1}{2} m_{i+1}\right)\right) .
\end{aligned}
$$

where $m_{i}$ is the slope coefficient of the "tilting" and is calculated with the following recursion

$$
m_{i}=m_{i+1}+\Delta t\left(D m_{i+1}^{2} J_{i}\right)
$$



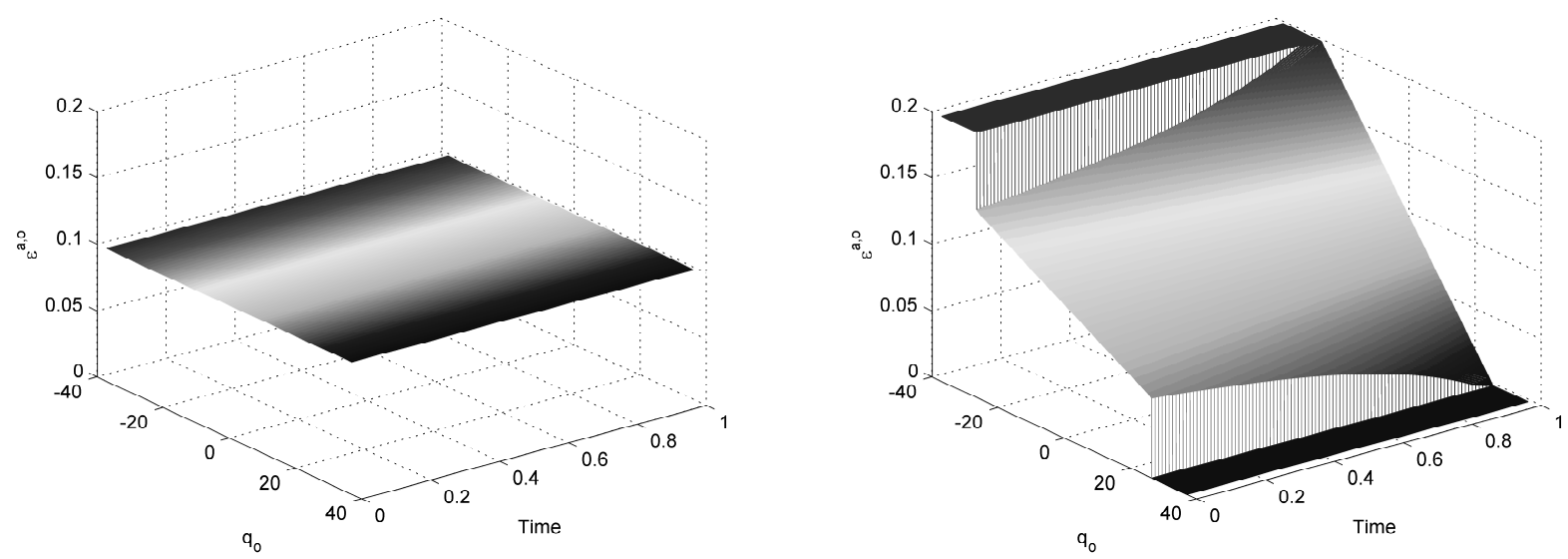

Figure 4: Optimal quoting policy for a long maturity option with $\alpha=0$ and $\alpha=0.00035$. In this scenario, the magnitude of $\alpha$ has a significant impact on the sensitivity of the quotes with respect to inventory. ( $\gamma=0.1, A=40, B=2000, C=40$, $D=200, S=100, \sigma_{0}=0.01, n=100, t_{0}=0, t_{n}=1, T=1.5, T_{\text {mat }}=400$.)
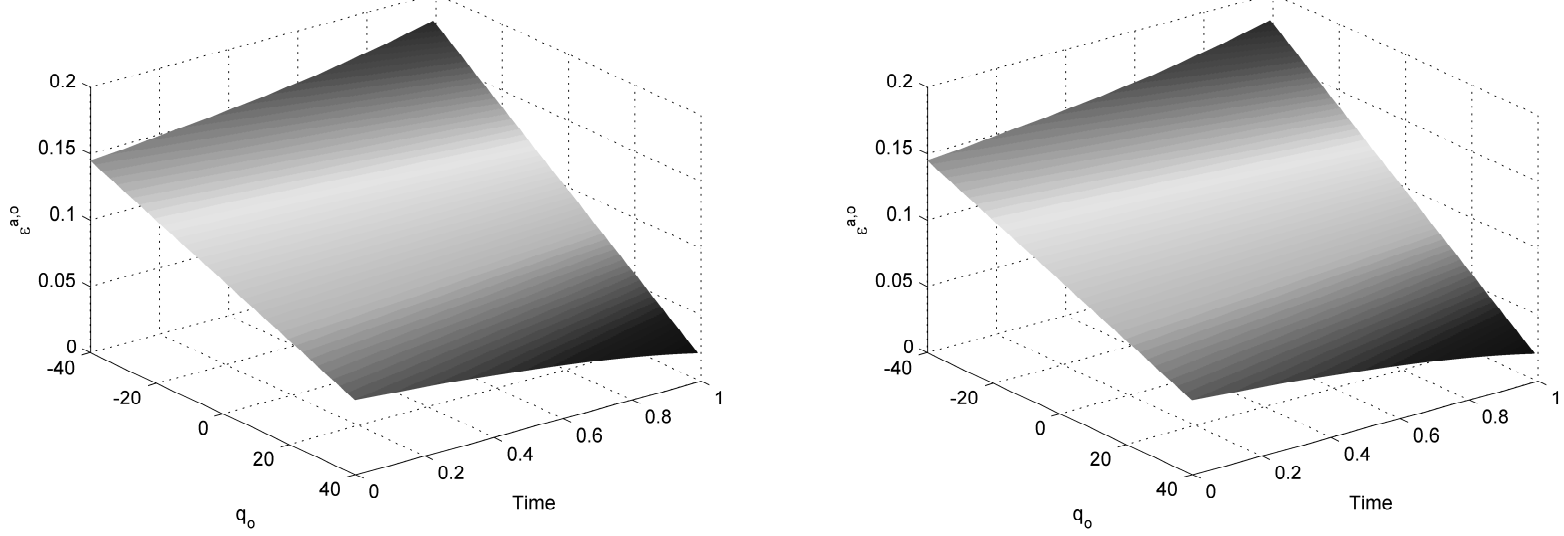

Figure 5: Optimal quoting policy for a very short maturity option with $\alpha=0$ and $\alpha=0.00035$. Gamma risk dominates in this scenario and the magnitude of $\alpha$ has no effect on the sensitivity of the quotes with respect to inventory. $(\gamma=0.1$, $A=40, B=2000, C=40, D=200, S=100, \sigma_{0}=0.01, n=100, t_{0}=0, t_{n}=1, T=1.5, T_{\text {mat }}=2$.)

with the terminal condition $m_{n}=-\gamma \sigma_{n}^{2} S^{2} \Gamma_{n}^{2}\left(T-t_{n}\right)\left(\frac{1}{2} \sigma_{n}^{2} S^{2}\left(T-t_{n}\right)+\alpha^{2} S^{2}\left(T_{m a t}-t_{n}\right)^{2}\right)$ and the auxiliary variable, $J_{i}=\left(\mathbb{1}_{\left\{0<\epsilon_{i}^{a, o}<\frac{C}{D}\right\}}+\mathbb{1}_{\left\{0<\epsilon_{i}^{b, o}<\frac{C}{D}\right\}}\right)$.

In Figure 4 and Figure 5, we illustrate the effect of the maturity, $T_{m a t}$, and the volatility of implied volatility, $\alpha$, on the option ask premiums. In Figure 5, we notice that for long maturity options, the magnitude of $\alpha$ has a significant impact on the sensitivity of the quotes with respect to inventory. This reflects the fact that Vega risk is more important than Gamma risk for long maturity options. However, when time-to-maturity is very short, Gamma risk dominates and the $\alpha$ parameter has virtually no 
effect on the optimal quoting policy (see Figure 5).

\section{Conclusion}

We approach the option pricing problem from the point of view of a market maker in European options. The dealer has control over bid and ask quotes and maximizes expected return, with a penalty that is proportional to the variance of the inventory value. The solution of this optimization problem illustrates that, after the purchase of an option lot, it is rational for option market makers to lower their bid and ask quotes. The extent to which their quotes are adjusted may be expressed in terms of the liquidity and incompleteness assumptions on the market.

In the complete market model, the dealer simultaneously updates bid and ask quotes on a European call option and costlessly adjusts the amount of underlying stock he holds. The mid prices of the stock and the option are related by the Black-Scholes formula. In this setting, every time the market maker sells or buys options, he is able to remove all risk by trading in the underlying. Consequently, he sets his quotes around the mid price in order to maximize return, regardless of his inventory position.

However, if the stock is illiquid, the dealer will be unable to trade continuously and may choose to make markets in the stock as well. In this setting, he will keep the net Delta of the inventory under control by updating his quotes on the stock and the option. Since we model the liquidity of the stock to be greater than that of the option, we find that the optimal strategy is to set a large bid-ask spread around the option mid price and move the stock quotes aggressively as the dealer's net Delta departs from zero. By varying the coefficient of risk aversion, we are able to numerically compute the efficient frontier of the dealer.

Finally, we model a dealer who delta-hedges the option inventory with the stock at every time step, but is subject to residual risks due to stochastic volatility and unhedgeable overnight moves in the stock price. These risks highlight the need to keep the Vega and Gamma of the dealer's inventory under control, and this is reflected in the dealer's quoting strategy. We illustrate this optimal quoting policy for options of varying maturity. We find that the dealer's net Gamma dominates the quoting policy for short maturity options, while the net Vega dominates the quoting policy for the long maturity options. 


\section{Appendix}

\section{A Proof of Theorem 1}

$\pi_{t}$ only appears in the variance term. Since $\mathbb{E}\left[I_{T}\right]=0, \operatorname{Var}\left[I_{T}\right]=\mathbb{E}\left[I_{T}^{2}\right]$. Using Ito isometry,

$$
\operatorname{Var}\left[I_{T}\right]=\mathbb{E}\left[I_{T}^{2}\right]=\mathbb{E}\left[\int_{0}^{T}\left(\sigma^{2} \pi_{t}^{2}+2 \sigma^{2} \pi_{t} S_{t} \Delta_{t} q_{t}^{o}+\sigma^{2}\left(q_{t}^{o}\right)^{2} \Delta_{t}^{2} S_{t}^{2}\right) d t\right]
$$

Taking first order conditions with respect to $\pi_{t}$, we obtain $\pi_{t}=-S_{t} \Delta_{t} q_{t}^{o}$.

Premium terms only appear in the expectation term. Since $\mathbb{E}\left[d N_{t}^{a, o}\right]=\lambda_{o}\left(\epsilon_{t}^{a, o}\right) d t$ and $\mathbb{E}\left[d N_{t}^{b, o}\right]=$ $\lambda_{o}\left(\epsilon_{t}^{b, o}\right) d t$, we obtain

$$
\mathbb{E}\left[Z_{T}\right]=\mathbb{E}\left[\int_{0}^{T}\left(\epsilon_{t}^{a, o} \lambda_{o}\left(\epsilon_{t}^{a, o}\right)+\epsilon_{t}^{b, o} \lambda_{o}\left(\epsilon_{t}^{b, o}\right)\right) d t\right] .
$$

This expression attains its maximum when the optimal premiums satisfy the following first order conditions:

$$
\begin{aligned}
& \lambda_{o}\left(\epsilon_{t}^{a, o}\right)+\epsilon_{t}^{a, o} \lambda_{o}^{\prime}\left(\epsilon_{t}^{a, o}\right)=0 \\
& \lambda_{o}\left(\epsilon_{t}^{b, o}\right)+\epsilon_{t}^{b, o} \lambda_{o}^{\prime}\left(\epsilon_{t}^{b, o}\right)=0 .
\end{aligned}
$$

Thus, $\epsilon_{t}^{a, o}=-\frac{\lambda_{o}\left(\epsilon_{t}^{a, o}\right)}{\lambda_{o}^{\prime}\left(\epsilon_{t}^{a, o}\right)}$ and $\epsilon_{t}^{b, o}=-\frac{\lambda_{o}\left(\epsilon_{t}^{b, o}\right)}{\lambda_{o}{ }^{b}\left(\epsilon_{t}^{b, o}\right)}$. Note that, for the existence of the maximizers, we need

$$
\epsilon \lambda_{o}^{\prime \prime}(\epsilon)+2 \lambda_{o}^{\prime}(\epsilon) \leq 0
$$

\section{B Proof of Theorem 2}

The expected wealth due to transactions conditional on the information at time $t_{n-1}$ equals

$$
\begin{aligned}
\mathbb{E}\left[Z_{T} \mid \mathcal{F}_{n-1}\right] & =Z_{n-1}+\mathbb{E}\left[\Delta Z_{n-1} \mid \mathcal{F}_{n-1}\right] \\
& =Z_{n-1}+\Delta t\left(\epsilon^{a, s} \lambda_{s}\left(\epsilon^{a, s}\right)+\epsilon^{b, s} \lambda_{s}\left(\epsilon^{b, s}\right)+\epsilon^{a, o} \lambda_{o}\left(\epsilon^{a, o}\right)+\epsilon^{b, o} \lambda_{o}\left(\epsilon^{b, o}\right)\right)
\end{aligned}
$$


where $\Delta t=t_{n}-t_{n-1}$. We then compute the variance terms using $S_{n-1}=S_{n}$ and $C_{n-1}=C_{n}$.

$$
\begin{aligned}
& \operatorname{Var}\left(\Delta I_{n} \mid \mathcal{F}_{n-1}\right)=\mathbb{E}\left[\operatorname{Var}\left(\Delta I_{n} \mid F_{n}\right) \mid F_{n-1}\right] \\
= & \mathbb{E}\left[\operatorname{Var}\left(q_{n}^{s}\left(S_{T}-S_{n}\right)+q_{n}^{o}\left(C_{T}-C_{n}\right) \mid F_{n}\right) \mid F_{n-1}\right] \\
= & \mathbb{E}\left[\left(q_{n}^{s} \sigma S_{n}+q_{n}^{o} \sigma S_{n} \Delta_{n}\right)^{2}\left(T-t_{n}\right) \mid F_{n-1}\right] \\
= & \lambda_{s}\left(\epsilon^{a, s}\right) \Delta t\left(\left(q_{n-1}^{s}-1\right) \sigma S_{n}+q_{n-1}^{o} \sigma S_{n} \Delta_{n}\right)^{2}\left(T-t_{n}\right)+\lambda_{s}\left(\epsilon^{b, s}\right) \Delta t\left(\left(q_{n-1}^{s}+1\right) \sigma S_{n}+q_{n-1}^{o} \sigma S_{n} \Delta_{n}\right)^{2}\left(T-t_{n}\right) \\
& +\lambda_{o}\left(\epsilon^{a, o}\right) \Delta t\left(q_{n-1}^{s} \sigma S_{n}+\left(q_{n-1}^{o}-1\right) \sigma S_{n} \Delta_{n}\right)^{2}\left(T-t_{n}\right)+\lambda_{s}\left(\epsilon^{b, o}\right) \Delta t\left(q_{n-1}^{s} \sigma S_{n}+\left(q_{n-1}^{o}+1\right) \sigma S_{n} \Delta_{n}\right)^{2}\left(T-t_{n}\right) \\
& +\left(1-\lambda_{s}\left(\epsilon^{a, s}\right) \Delta t-\lambda_{s}\left(\epsilon^{b, s}\right) \Delta t-\lambda_{o}\left(\epsilon^{a, o}\right) \Delta t-\lambda_{o}\left(\epsilon^{b, o}\right) \Delta t\right)\left(\left(q_{n-1}^{s} \sigma S_{n}+q_{n-1}^{o} \sigma S_{n} \Delta_{n}\right)\right)^{2}\left(T-t_{n}\right)
\end{aligned}
$$

which equals after cancelations:

$$
\begin{aligned}
& =\left(T-t_{n}\right)\left(\lambda_{s}\left(\epsilon^{a, s}\right) \Delta t\left(\left(-2 q_{n-1}^{s}+1\right) \sigma^{2} S_{n}^{2}-2 q_{n-1}^{o} \sigma^{2} S_{n}^{2} \Delta_{n}\right)+\lambda_{s}\left(\epsilon^{b, s}\right) \Delta t\left(\left(2 q_{n-1}^{s}+1\right) \sigma^{2} S_{n}^{2}+2 q_{n-1}^{o} \sigma^{2} S_{n}^{2} \Delta_{n}\right)\right. \\
& +\lambda_{o}\left(\epsilon^{a, o}\right) \Delta t\left(\left(-2 q_{n-1}^{o}+1\right) \sigma^{2} S_{n}^{2} \Delta_{n}^{2}-2 q_{n-1}^{s} \sigma^{2} S_{n}^{2} \Delta_{n}\right)+\lambda_{o}\left(\epsilon^{b, o}\right) \Delta t\left(\left(2 q_{n-1}^{o}+1\right) \sigma^{2} S_{n}^{2} \Delta_{n}^{2}+2 q_{n-1}^{s} \sigma^{2} S_{n}^{2} \Delta_{n}\right) \\
& \left.+\left(\left(q_{n-1}^{s}\right)^{2} \sigma^{2} S_{n}^{2}+\left(q_{n-1}^{o}\right)^{2} \sigma^{2} S_{n}^{2} \Delta_{n}^{2}+2 q_{n-1}^{s} q_{n-1}^{o} \sigma^{2} S_{n}^{2} \Delta_{n}\right)\right)
\end{aligned}
$$

Using the above formulas, $v\left(X_{n-1}, S_{n-1}, q_{n-1}^{s}, q_{n-1}^{o}, t_{n-1}\right)$ then equals:

$$
\begin{aligned}
& \max _{\substack{a, s \\
\epsilon_{n-1}, \epsilon_{n-1}^{b, s}, \epsilon_{n-1}^{a, o}, \epsilon_{n-1}^{b, o}}}\left\{\left(Y_{n-1}+\Delta t\left(\epsilon^{a, s} \lambda_{s}\left(\epsilon^{a, s}\right)+\epsilon^{b, s} \lambda_{s}\left(\epsilon^{b, s}\right)+\epsilon^{a, o} \lambda_{o}\left(\epsilon^{a, o}\right)+\epsilon^{b, o} \lambda_{o}\left(\epsilon^{b, o}\right)\right)\right)\right. \\
& -\gamma\left(T-t_{n}\right)\left(\lambda_{s}\left(\epsilon^{a, s}\right) \Delta t\left(\left(-2 q_{n-1}^{s}+1\right) \sigma^{2} S_{n}^{2}-2 q_{n-1}^{o} \sigma^{2} S_{n}^{2} \Delta_{n}\right)+\lambda_{s}\left(\epsilon^{b, s}\right) \Delta t\left(\left(2 q_{n-1}^{s}+1\right) \sigma^{2} S_{n}^{2}+2 q_{n-1}^{o} \sigma^{2} S_{n}^{2} \Delta_{n}\right)\right. \\
& +\lambda_{o}\left(\epsilon^{a, o}\right) \Delta t\left(\left(-2 q_{n-1}^{o}+1\right) \sigma^{2} S_{n}^{2} \Delta_{n}^{2}-2 q_{n-1}^{s} \sigma^{2} S_{n}^{2} \Delta_{n}\right)+\lambda_{o}\left(\epsilon^{b, o}\right) \Delta t\left(\left(2 q_{n-1}^{o}+1\right) \sigma^{2} S_{n}^{2} \Delta_{n}^{2}+2 q_{n-1}^{s} \sigma^{2} S_{n}^{2} \Delta_{n}\right) \\
& \left.\left.+\left(\left(q_{n-1}^{s}\right)^{2} \sigma^{2} S_{n}^{2}+\left(q_{n-1}^{o}\right)^{2} \sigma^{2} S_{n}^{2} \Delta_{n}^{2}+2 q_{n-1}^{s} q_{n-1}^{o} \sigma^{2} S_{n}^{2} \Delta_{n}\right)\right)\right\}
\end{aligned}
$$

By taking the first order conditions, we can solve for each bid and ask quote for both the option and the stock:

$$
\begin{aligned}
\epsilon_{n-1}^{a, s} & =\max \left(0, \min \left(\frac{A}{B}, \frac{A}{2 B}-\gamma \sigma^{2}\left(T-t_{n}\right) S_{n}^{2}\left(q_{n-1}^{s}+q_{n-1}^{o} \Delta_{n}-\frac{1}{2}\right)\right)\right) \\
\epsilon_{n-1}^{b, s} & =\max \left(0, \min \left(\frac{A}{B}, \frac{A}{2 B}+\gamma \sigma^{2}\left(T-t_{n}\right) S_{n}^{2}\left(q_{n-1}^{s}+q_{n-1}^{o} \Delta_{n}+\frac{1}{2}\right)\right)\right) \\
\epsilon_{n-1}^{a, o} & =\max \left(0, \min \left(\frac{C}{D}, \frac{C}{2 D}-\gamma \sigma^{2}\left(T-t_{n}\right) S_{n}^{2} \Delta_{n}\left(q_{n-1}^{s}+q_{n-1}^{o} \Delta_{n}-\frac{1}{2} \Delta_{n}\right)\right)\right) \\
\epsilon_{n-1}^{b, o} & =\max \left(0, \min \left(\frac{C}{D}, \frac{C}{2 D}+\gamma \sigma^{2}\left(T-t_{n}\right) S_{n}^{2} \Delta_{n}\left(q_{n-1}^{s}+q_{n-1}^{o} \Delta_{n}+\frac{1}{2} \Delta_{n}\right)\right)\right)
\end{aligned}
$$




\section{Proof of Theorem 3}

We need the following lemmas to prove Theorem 3 .

Lemma 6. Let $I_{k}=\left(\mathbb{1}_{\left\{0<\epsilon_{k}^{a, s}<\frac{A}{B}\right\}}+\mathbb{1}_{\left\{0<\epsilon_{k}^{b, s}<\frac{A}{B}\right\}}\right)$ and $J_{k}=\left(\mathbb{1}_{\left\{0<\epsilon_{k}^{a, o}<\frac{C}{D}\right\}}+\mathbb{1}_{\left\{0<\epsilon_{k}^{b, o}<\frac{C}{D}\right\}}\right)$. Then, $v_{i}\left(Z_{i}, q_{i}^{s}, q_{i}^{o}\right)=Z_{i}+w_{i}\left(q_{i}^{s}, q_{i}^{o}\right)$ and $w_{i}\left(q_{i}^{s}, q_{i}^{o}\right)$ is a non-linear function of inventory in the form

$$
a_{i}\left(q_{i}^{s}\right)^{2}+b_{i}\left(q_{i}^{o}\right)^{2}+c_{i} q_{i}^{s} q_{i}^{o}+d_{i} q_{i}^{s}+e_{i} q_{i}^{o}+f_{i}
$$

For $k<n$, we have

$$
\begin{aligned}
a_{k}= & a_{k+1}+\Delta t\left(B a_{k+1}^{2} I_{k}+\frac{1}{4} D c_{k+1}^{2} J_{k}\right) \\
b_{k}= & b_{k+1}+\Delta t\left(D b_{k+1}^{2} J_{k}+\frac{1}{4} B c_{k+1}^{2} I_{k}\right) \\
c_{k}= & c_{k+1}+\Delta t\left(B a_{k+1} c_{k+1} I_{k}+D b_{k+1} c_{k+1} J_{k}\right) \\
d_{k}= & d_{k+1}+\Delta t\left(B a_{k+1} d_{k+1} I_{k}+\frac{1}{4} D c_{k+1} e_{k+1} J_{k}\right) \\
e_{k}= & e_{k+1}+\Delta t\left(D b_{k+1} e_{k+1} J_{k}+\frac{1}{4} B c_{k+1} d_{k+1} I_{k}\right) \\
f_{k}= & f_{k+1}+\Delta t\left\{\left(\frac{A^{2}}{4 B}+\frac{1}{4} B\left(a_{k+1}^{2}+d_{k+1}^{2}\right)+\frac{1}{2} A a_{k+1}\right) I_{k}+\left(\frac{C^{2}}{4 D}+\frac{1}{4} D\left(b_{k+1}^{2}+e_{k+1}^{2}\right)+\frac{1}{2} C b_{k+1}\right) J_{k}\right. \\
& \left.-A a_{k+1}\left(\mathbb{1}_{\left\{\epsilon_{k}^{a, s}=0\right\}}+\mathbb{1}_{\left\{\epsilon_{k}^{b, s}=0\right\}}\right)-C b_{k+1}\left(\mathbb{1}_{\left\{\epsilon_{k}^{a, o}=0\right\}}+\mathbb{1}_{\left\{\epsilon_{k}^{b, o}=0\right\}}\right)\right\}
\end{aligned}
$$

and

$$
\begin{aligned}
\epsilon_{k}^{a, s} & =\max \left(0, \min \left(\frac{A}{B}, \frac{A}{2 B}+a_{k+1} q_{k}^{s}+\frac{1}{2} c_{k+1} q_{k}^{o}-\frac{1}{2} a_{k+1}+\frac{1}{2} d_{k+1}\right)\right) \\
\epsilon_{k}^{b, s} & =\max \left(0, \min \left(\frac{A}{B}, \frac{A}{2 B}-a_{k+1} q_{k}^{s}-\frac{1}{2} c_{k+1} q_{k}^{o}-\frac{1}{2} a_{k+1}-\frac{1}{2} d_{k+1}\right)\right) \\
\epsilon_{k}^{a, o} & =\max \left(0, \min \left(\frac{C}{D}, \frac{C}{2 D}+b_{k+1} q_{k}^{o}+\frac{1}{2} c_{k+1} q_{k}^{s}-\frac{1}{2} b_{k+1}+\frac{1}{2} e_{k+1}\right)\right) \\
\epsilon_{k}^{b, o} & =\max \left(0, \min \left(\frac{C}{D}, \frac{C}{2 D}-b_{k+1} q_{k}^{o}-\frac{1}{2} c_{k+1} q_{k}^{s}-\frac{1}{2} b_{k+1}-\frac{1}{2} e_{k+1}\right)\right)
\end{aligned}
$$

Proof. This lemma can be proven by induction.

Base case: If $i=n$, then

$$
v_{n}\left(Z_{n}, q_{n}^{s}, q_{n}^{o}\right)=Z_{n}-\gamma \sigma^{2} S_{n}^{2}\left(T-t_{n}\right)\left(q_{n}^{s}+q_{n}^{o} \Delta_{n}\right)^{2}
$$


which implies that

$$
\begin{aligned}
& a_{n}=-\gamma \sigma^{2} S^{2}\left(T-t_{n}\right) \\
& b_{n}=-\gamma \sigma^{2} S^{2} \Delta_{n}^{2}\left(T-t_{n}\right) \\
& c_{n}=-2 \gamma \sigma^{2} S^{2} \Delta_{n}\left(T-t_{n}\right) \\
& d_{n}=0 \\
& e_{n}=0 .
\end{aligned}
$$

Inductive step: Suppose Lemma 6 holds for $i=k+1$ where $0<k+1 \leq n$. Then for $i=k$, we use

$$
v_{k}\left(Z_{k}, q_{k}^{s}, q_{k}^{o}\right)=\max _{\epsilon_{k}^{a, s}, \epsilon_{k}^{b, s}, \epsilon_{k}^{a, o}, \epsilon_{k}^{b, o}} \mathbb{E}\left[v_{k+1}\left(Z_{k+1}, q_{k+1}^{s}, q_{k+1}^{o}\right) \mid \mathcal{F}_{k}\right]
$$

Using induction hypothesis, $v_{k}\left(Z_{k}, q_{k}^{s}, q_{k}^{o}\right)$ equals

$$
\begin{aligned}
= & \max _{\epsilon_{k}^{a, s}, \epsilon_{k}^{b, s}, \epsilon_{k}^{a, o}, \epsilon_{k}^{b, o}} \mathbb{E}\left[Z_{k+1}+a_{k+1}\left(q_{k}^{s}\right)^{2}+b_{k+1}\left(q_{k}^{o}\right)^{2}+c_{k+1} q_{k}^{s} q_{k}^{o}+d_{k+1} q_{k}^{s}+e_{k+1} q_{k}^{o}+f_{k+1} \mid \mathcal{F}_{k}\right] \\
= & Z_{k}+\left(\epsilon_{k}^{a, s} \lambda_{s}\left(\epsilon_{k}^{a, s}\right)+\epsilon_{k}^{b, s} \lambda_{s}\left(\epsilon_{k}^{b, s}\right)+\epsilon_{k}^{a, o} \lambda_{o}\left(\epsilon_{k}^{a, o}\right)+\epsilon_{k}^{b, o} \lambda_{o}\left(\epsilon_{k}^{b, o}\right)\right) \Delta t \\
& +\lambda_{s}\left(\epsilon_{k}^{a, s}\right) \Delta t\left(a_{k+1}\left(q_{k}^{s}-1\right)^{2}+b_{k+1}\left(q_{k}^{o}\right)^{2}+c_{k+1}\left(q_{k}^{s}-1\right) q_{k}^{o}+d_{k+1}\left(q_{k}^{s}-1\right)+e_{k+1} q_{k}^{o}+f_{k+1}\right) \\
& +\lambda_{s}\left(\epsilon_{k}^{b, s}\right) \Delta t\left(a_{k+1}\left(q_{k}^{s}+1\right)^{2}+b_{k+1}\left(q_{k}^{o}\right)^{2}+c_{k+1}\left(q_{k}^{s}+1\right) q_{k}^{o}+d_{k+1}\left(q_{k}^{s}+1\right)+e_{k+1} q_{k}^{o}+f_{k+1}\right) \\
& +\lambda_{o}\left(\epsilon_{k}^{a, o}\right) \Delta t\left(a_{k+1}\left(q_{k}^{s}\right)^{2}+b_{k+1}\left(q_{k}^{o}-1\right)^{2}+c_{k+1} q_{k}^{s}\left(q_{k}^{o}-1\right)+d_{k+1} q_{k}^{s}+e_{k+1}\left(q_{k}^{o}-1\right)+f_{k+1}\right) \\
& +\lambda_{s}\left(\epsilon_{k}^{b, o}\right) \Delta t\left(a_{k+1}\left(q_{k}^{s}\right)^{2}+b_{k+1}\left(q_{k}^{o}+1\right)^{2}+c_{k+1} q_{k}^{s}\left(q_{k}^{o}+1\right)+d_{k+1} q_{k}^{s}+e_{k+1}\left(q_{k}^{o}+1\right)+f_{k+1}\right) \\
& +\left(1-\lambda_{s}\left(\epsilon_{k}^{a, s}\right) \Delta t-\lambda_{s}\left(\epsilon_{k}^{b, s}\right) \Delta t-\lambda_{o}\left(\epsilon_{k}^{a, o}\right) \Delta t-\lambda_{o}\left(\epsilon_{k}^{b, o}\right) \Delta t\right)\left(a_{k+1}\left(q_{k}^{s}\right)^{2}+b_{k+1}\left(q_{k}^{o}\right)^{2}\right. \\
& \left.+c_{k+1} q_{k}^{s} q_{k}^{o}+d_{k+1} q_{k}^{s}+e_{k+1} q_{k}^{o}+f_{k+1}\right) .
\end{aligned}
$$

Finally, with some algebraic manipulation, we obtain

$$
\begin{aligned}
= & Z_{k}+\left(\epsilon_{k}^{a, s} \lambda_{s}\left(\epsilon_{k}^{a, s}\right)+\epsilon_{k}^{b, s} \lambda_{s}\left(\epsilon_{k}^{b, s}\right)+\epsilon_{k}^{a, o} \lambda_{o}\left(\epsilon_{k}^{a, o}\right)+\epsilon_{k}^{b, o} \lambda_{o}\left(\epsilon_{k}^{b, o}\right)\right) \Delta t \\
& +\lambda_{s}\left(\epsilon_{k}^{a, s}\right) \Delta t\left(a_{k+1}\left(-2 q_{k}^{s}+1\right)-c_{k+1} q_{k}^{o}-d_{k+1}\right) \\
& +\lambda_{s}\left(\epsilon_{k}^{b, s}\right) \Delta t\left(a_{k+1}\left(2 q_{k}^{s}+1\right)+c_{k+1} q_{k}^{o}+d_{k+1}\right)+\lambda_{o}\left(\epsilon_{k}^{a, o}\right) \Delta t\left(b_{k+1}\left(-2 q_{k}^{o}+1\right)-c_{k+1} q_{k}^{s}-e_{k+1}\right) \\
& +\lambda_{s}\left(\epsilon_{k}^{b, o}\right) \Delta t\left(b_{k+1}\left(2 q_{k}^{o}+1\right)+c_{k+1} q_{k}^{s}+e_{k+1}\right)\left(a_{k+1}\left(q_{k}^{s}\right)^{2}+b_{k+1}\left(q_{k}^{o}\right)^{2}+c_{k+1} q_{k}^{s} q_{k}^{o}+d_{k+1} q_{k}^{s}+e_{k+1} q_{k}^{o}+f_{k+1}\right)
\end{aligned}
$$


If we take the first order conditions in the following terms,

$$
\begin{aligned}
& \epsilon_{k}^{a, s}\left(A-B \epsilon_{k}^{a, s}\right)+\left(A-B \epsilon_{k}^{a, s}\right)\left(-2 a_{k+1} q_{k}^{s}-c_{k+1} q_{k}^{o}+a_{k+1}-d_{k+1}\right) \\
& +\epsilon_{k}^{a, s}\left(A-B \epsilon_{k}^{b, s}\right)+\left(A-B \epsilon_{k}^{b, s}\right)\left(2 a_{k+1} q_{k}^{s}+c_{k+1} q_{k}^{o}+a_{k+1}+d_{k+1}\right) \\
& +\epsilon_{k}^{a, o}\left(C-D \epsilon_{k}^{a, o}\right)+\left(C-D \epsilon_{k}^{a, o}\right)\left(-2 b_{k+1} q_{k}^{o}-c_{k+1} q_{k}^{s}+b_{k+1}-e_{k+1}\right) \\
& +\epsilon_{k}^{b, o}\left(C-D \epsilon_{k}^{b, o}\right)+\left(C-D \epsilon_{k}^{b, o}\right)\left(2 b_{k+1} q_{k}^{o}+c_{k+1} q_{k}^{s}+b_{k+1}+e_{k+1}\right)
\end{aligned}
$$

we obtain the optimal premiums for each trading session:

$$
\begin{aligned}
\epsilon_{k}^{a, s} & =\max \left(0, \min \left(\frac{A}{B}, \frac{A}{2 B}+a_{k+1} q_{k}^{s}+\frac{1}{2} c_{k+1} q_{k}^{o}-\frac{1}{2} a_{k+1}+\frac{1}{2} d_{k+1}\right)\right) \\
\epsilon_{k}^{b, s} & =\max \left(0, \min \left(\frac{A}{B}, \frac{A}{2 B}-a_{k+1} q_{k}^{s}-\frac{1}{2} c_{k+1} q_{k}^{o}-\frac{1}{2} a_{k+1}-\frac{1}{2} d_{k+1}\right)\right) \\
\epsilon_{k}^{a, o} & =\max \left(0, \min \left(\frac{C}{D}, \frac{C}{2 D}+b_{k+1} q_{k}^{o}+\frac{1}{2} c_{k+1} q_{k}^{s}-\frac{1}{2} b_{k+1}+\frac{1}{2} e_{k+1}\right)\right) \\
\epsilon_{k}^{b, o} & =\max \left(0, \min \left(\frac{C}{D}, \frac{C}{2 D}-b_{k+1} q_{k}^{o}-\frac{1}{2} c_{k+1} q_{k}^{s}-\frac{1}{2} b_{k+1}-\frac{1}{2} e_{k+1}\right)\right)
\end{aligned}
$$

If we substitute these optimal premiums back into the value function, we obtain

$$
\begin{aligned}
v_{k}\left(Z_{k}, q_{k}^{s}, q_{k}^{o}\right)= & Z_{k}+\mathbb{1}_{\left\{0<\epsilon_{k}^{a, s}<\frac{A}{B}\right\}} B \Delta t\left(\frac{A}{2 B}-a_{k+1} q_{k}^{s}-\frac{1}{2} c_{k+1} q_{k}^{o}+\frac{1}{2} a_{k+1}-\frac{1}{2} d_{k+1}\right)^{2} \\
& -\mathbb{1}_{\left\{\epsilon_{k}^{a, s}=0\right\}} 2 A \Delta t\left(-a_{k+1} q_{k}^{s}-\frac{1}{2} c_{k+1} q_{k}^{o}+\frac{1}{2} a_{k+1}-\frac{1}{2} d_{k+1}\right) \\
& +\mathbb{1}_{\left\{0<\epsilon_{k}^{b, s}<\frac{A}{B}\right\}} B \Delta t\left(\frac{A}{2 B}+a_{k+1} q_{k}^{s}+\frac{1}{2} c_{k+1} q_{k}^{o}+\frac{1}{2} a_{k+1}+\frac{1}{2} d_{k+1}\right)^{2} \\
& -\mathbb{1}_{\left\{\epsilon_{k}^{b, s}=0\right\}} 2 A \Delta t\left(a_{k+1} q_{k}^{s}+\frac{1}{2} c_{k+1} q_{k}^{o}+\frac{1}{2} a_{k+1}+\frac{1}{2} d_{k+1}\right) \\
& +\mathbb{1}_{\left\{0<\epsilon_{k}^{a, o}<\frac{C}{D}\right\}} D \Delta t\left(\frac{C}{2 D}-b_{k+1} q_{k}^{o}-\frac{1}{2} c_{k+1} q_{k}^{s}+\frac{1}{2} b_{k+1}-\frac{1}{2} e_{k+1}\right)^{2} \\
& -\mathbb{1}_{\left\{\epsilon_{k}^{a, o}=0\right\}} 2 C \Delta t\left(-b_{k+1} q_{k}^{o}-\frac{1}{2} c_{k+1} q_{k}^{s}+\frac{1}{2} b_{k+1}-\frac{1}{2} e_{k+1}\right) \\
& +\mathbb{1}_{\left\{0<\epsilon_{k}^{b, o}<\frac{C}{D}\right\}} D \Delta t\left(\frac{C}{2 D}+b_{k+1} q_{k}^{o}+\frac{1}{2} c_{k+1} q_{k}^{s}+\frac{1}{2} b_{k+1}+\frac{1}{2} e_{k+1}\right)^{2} \\
& -\mathbb{1}_{\left\{\epsilon_{k}^{b, o}=0\right\}} 2 C \Delta t\left(b_{k+1} q_{k}^{o}+\frac{1}{2} c_{k+1} q_{k}^{s}+\frac{1}{2} b_{k+1}+\frac{1}{2} e_{k+1}\right) \\
& +\left[a_{k+1}\left(q_{k}^{s}\right)^{2}+b_{k+1}\left(q_{k}^{o}\right)^{2}+c_{k+1} q_{k}^{s} q_{k}^{o}+d_{k+1} q_{k}^{s}+e_{k+1} q_{k}^{o}+f_{k+1}\right]
\end{aligned}
$$


This shows that $v_{k}\left(Z_{k}, q_{k}^{s}, q_{k}^{o}\right)$ is a non-linear function of inventory with the following coefficients.

$$
\begin{aligned}
a_{k}= & a_{k+1}+\Delta t\left(B a_{k+1}^{2} I_{k}+\frac{1}{4} D c_{k+1}^{2} J_{k}\right) \\
b_{k}= & b_{k+1}+\Delta t\left(D b_{k+1}^{2} J_{k}+\frac{1}{4} B c_{k+1}^{2} I_{k}\right) \\
c_{k}= & c_{k+1}+\Delta t\left(B a_{k+1} c_{k+1} I_{k}+D b_{k+1} c_{k+1} J_{k}\right) \\
d_{k}= & d_{k+1}+\Delta t\left(B a_{k+1} d_{k+1} I_{k}+\frac{1}{4} D c_{k+1} e_{k+1} J_{k}\right) \\
e_{k}= & e_{k+1}+\Delta t\left(D b_{k+1} e_{k+1} J_{k}+\frac{1}{4} B c_{k+1} d_{k+1} I_{k}\right) \\
f_{k}= & f_{k+1}+\Delta t\left\{\left(\frac{A^{2}}{4 B}+\frac{1}{4} B\left(a_{k+1}^{2}+d_{k+1}^{2}\right)+\frac{1}{2} A a_{k+1}\right) I_{k}+\left(\frac{C^{2}}{4 D}+\frac{1}{4} D\left(b_{k+1}^{2}+e_{k+1}^{2}\right)+\frac{1}{2} C b_{k+1}\right) J_{k}\right. \\
& \left.-A a_{k+1}\left(\mathbb{1}_{\left\{\epsilon_{k}^{a, s}=0\right\}}+\mathbb{1}_{\left\{\epsilon_{k}^{b, s}=0\right\}}\right)-C b_{k+1}\left(\mathbb{1}_{\left\{\epsilon_{k}^{a, o}=0\right\}}+\mathbb{1}_{\left\{\epsilon_{k}^{b, o}=0\right\}}\right)\right\}
\end{aligned}
$$

Lemma 7. The coefficients $b_{i}$ and $c_{i}$ can be written in terms of $a_{i}$ such that $b_{i}=a_{i} \Delta_{n}^{2}$ and $c_{i}=2 a_{i} \Delta_{n}$. Furthermore, due to the terminal conditions, $d_{i}=e_{i}=0$.

Proof. This lemma can also be proven by induction.

Base case: If $i=n$, then

$$
\begin{aligned}
& a_{n}=-\gamma \sigma^{2} S^{2}\left(T-t_{n}\right) \\
& b_{n}=a_{n} \Delta_{n}^{2} \\
& c_{n}=2 a_{n} \Delta_{n} \\
& d_{n}=0 \\
& e_{n}=0 .
\end{aligned}
$$

Inductive step: Suppose Lemma 7 holds for $i=k+1$ where $0<k+1 \leq n$. Then for $i=k$, we use 
the following identities from Lemma 6 .

$$
\begin{aligned}
& b_{k}=b_{k+1}+\Delta t\left(D b_{k+1}^{2} J_{k}+\frac{1}{4} B c_{k+1}^{2} I_{k}\right) \\
& c_{k}=c_{k+1}+\Delta t\left(B a_{k+1} c_{k+1} I_{k}+D b_{k+1} c_{k+1} J_{k}\right) \\
& d_{k}=d_{k+1}+\Delta t\left(B a_{k+1} d_{k+1} I_{k}+\frac{1}{4} D c_{k+1} e_{k+1} J_{k}\right) \\
& e_{k}=e_{k+1}+\Delta t\left(D b_{k+1} e_{k+1} I_{k}+\frac{1}{4} B c_{k+1} d_{k+1} J_{k}\right)
\end{aligned}
$$

Using induction hypothesis,

$$
\begin{aligned}
b_{k} & =a_{k+1} \Delta_{n}^{2}+\Delta t\left(J_{k} D\left(a_{k+1} \Delta_{n}^{2}\right)^{2}+\frac{1}{4} I_{k} B\left(2 a_{k+1} \Delta_{n}\right)^{2}\right) \\
& =\Delta_{n}^{2}\left(a_{k+1}+\Delta t\left(I_{k} B a_{k+1}^{2}+\frac{1}{4} J_{k} D c_{k+1}^{2}\right)\right) \\
& =\Delta_{n}^{2} a_{k} \\
c_{k} & =a_{k+1} \Delta_{n}+\Delta t\left(I_{k} B a_{k+1} a_{k+1} \Delta_{n}+J_{k} D a_{k+1} \Delta_{n}^{2} a_{k+1} \Delta_{n}\right) \\
& =2 \Delta_{n}\left(a_{k+1}+\Delta t\left(I_{k} B a_{k+1}^{2}+\frac{1}{4} J_{k} c_{k+1}^{2}\right)\right) \\
& =2 \Delta_{n} a_{k} \\
d_{k} & =d_{k+1}+\Delta t\left(I_{k} B a_{k+1} d_{k+1}+\frac{1}{4} J_{k} D c_{k+1} e_{k+1}\right) \\
& =0+\Delta t\left(I_{k} B a_{k+1} 0+\frac{1}{4} J_{k} D c_{k+1} 0\right) \\
& =0 \\
e_{k} & =e_{k+1}+\Delta t\left(J_{k} D b_{k+1} e_{k+1}+\frac{1}{4} I_{k} B c_{k+1} d_{k+1}\right) \\
& =0+\Delta t\left(J_{k} D b_{k+1} 0+\frac{1}{4} I_{k} c_{k+1} 0\right) \\
& =0
\end{aligned}
$$


By using Lemma 6 and Lemma 7, and letting $m_{i}=a_{i}$, we get

$$
\begin{aligned}
m_{i} & =m_{i+1}+\Delta t\left(B m_{i+1}^{2} I_{i}+D \Delta_{n}^{2} m_{i+1}^{2} J_{i}\right) \\
f_{i} & =f_{i+1}+\Delta t\left\{\left(\frac{A^{2}}{4 B}+\frac{1}{4} B m_{i+1}^{2}+\frac{1}{2} A m_{i+1}\right) I_{i}+\left(\frac{C^{2}}{4 D}+\frac{1}{4} D m_{i+1}^{2} \Delta_{n}^{4}+\frac{1}{2} C m_{i+1} \Delta_{n}^{2}\right) J_{i}\right. \\
& \left.-A m_{i+1}\left(\mathbb{1}_{\left\{\epsilon_{i}^{a, s}=0\right\}}+\mathbb{1}_{\left\{\epsilon_{i}^{b, s}=0\right\}}\right)-C m_{i+1} \Delta_{n}^{2}\left(\mathbb{1}_{\left\{\epsilon_{i}^{b, o}=0\right\}}+\mathbb{1}_{\left\{\epsilon_{i}^{b, o}=0\right\}}\right)\right\} .
\end{aligned}
$$

As a result, the optimal bid and ask premiums at time $t_{i}$ are given by

$$
\begin{aligned}
\epsilon_{i}^{a, s} & =\max \left(0, \min \left(\frac{A}{B}, \frac{A}{2 B}+m_{i+1}\left(q_{i}^{s}+\Delta_{n} q_{i}^{o}-\frac{1}{2}\right)\right)\right) \\
\epsilon_{i}^{b, s} & =\max \left(0, \min \left(\frac{A}{B}, \frac{A}{2 B}-m_{i+1}\left(q_{i}^{s}+\Delta_{n} q_{i}^{o}+\frac{1}{2}\right)\right)\right) \\
\epsilon_{i}^{a, o} & =\max \left(0, \min \left(\frac{C}{D}, \frac{C}{2 D}+m_{i+1} \Delta_{n}\left(q_{i}^{s}+\Delta_{n} q_{i}^{o}-\frac{1}{2} \Delta_{n}\right)\right)\right) \\
\epsilon_{i}^{a, o} & =\max \left(0, \min \left(\frac{C}{D}, \frac{C}{2 D}-m_{i+1} \Delta_{n}\left(q_{i}^{s}+\Delta_{n} q_{i}^{o}+\frac{1}{2} \Delta_{n}\right)\right)\right)
\end{aligned}
$$

\section{Proof of Theorem 4 and Theorem 5}

The proofs for these theorems are identically same with Theorem 2 and Theorem 3 respectively except that there are no stock premiums. Proofs can be obtained by contacting the authors. 


\section{References}

[1] Almgren, R. and Chriss, N. (2001): "Optimal Execution of Portfolio Transactions", Journal of Risk, 3: 5-39.

[2] Boyle, P. and Emanuel, D. (1980): "Discretely Adjusted Option Hedges", Journal of Financial Economics, 8: 259-282.

[3] Cont, R. and da Fonseca, J. (2002): "Dynamics of Implied Volatility Surfaces", Quantitative Finance, 2: 45-60.

[4] Davis, M. H. A., Panas, V. G., and Zariphopoulou, T. (1993): "European option pricing with transaction costs", SIAM Journal on Control and Optimization, 31(2): 470493.

[5] Engle, R. and Ferstenberg, R. (2006): "Execution Risk", Working paper.

[6] Garleanu, N., Pedersen, L. H. and Poteshman, A. M. (2006): "Demand Based Option Pricing", Working Paper, Wharton School of Business.

[7] Hasbrouck, J. (2007): Empirical Market Microstructure. New York: Oxford University Press.

[8] Ho, T. S. Y. and Macris, R. G. (1984): "Dealer Bid-Ask Quotes and Transaction Prices: An Empirical Study of Some AMEX Options", Journal of Finance, 39(1), 23-45.

[9] Ho, T. S. Y. and Stoll H. R. (1981): "Optimal Dealer Pricing under Transactions and Return Uncertainty", Journal of Financial Economics, 9, 47-73.

[10] Jameson, M. and Wilhelm, W. (1992): "Market Making in the Options Markets and the Costs of Discrete Hedge Rebalancing", Journal of Finance, 47, 765-779.

[11] Minina, V. and Vellekoop, M. (2008): “A Risk Reserve Model for Hedging in Incomplete Markets Rebalancing", Working paper.

[12] Schönbucher, P. J. (1999): "A Market Model for Stochastic Implied Volatility", Philosophical Transactions: Mathematical, Physical and Engineering Sciences, 357(1758), 2071-2092. 